\title{
Magnesium Alloy Stent Expansion Behavior Simulated by Finite Element Method
}

\author{
Muhammad Iqbal Sabir ${ }^{1, a}$, Erbao Liu ${ }^{1, b}$, Zhen $\mathrm{Li}^{1, \mathrm{c}}$, Yufeng Zheng ${ }^{2, \mathrm{~d}}$, \\ Li Li ${ }^{1,2, e, *}$ \\ ${ }^{1}$ Center for Biomedical Materials and Engineering, Harbin Engineering University, China \\ ${ }^{2}$ State Key Laboratory of Turbulence and Complex Systems, Peking University, China \\ aiqbalsabir@hotmail.com, bliutong_05@163.com, 'lz_heu@hrbeu.edu.cn , \\ dyfzheng@pku.edu.cn, e,*lili_heu@hrbeu.edu.cn
}

\begin{abstract}
Keywords: Finite Element Analysis, Mechanical Characteristic, Transient Non-uniform, Biodegradable coronary Stent, Magnesium Alloy.
\end{abstract}

\begin{abstract}
Biodegradable magnesium alloy stents have gained increasing interest in the past years due to their potential prospect. Magnesium alloy is brittle compared with stainless steel. This means it has less elongation than other stent materials and it may cause strut break under large deformation. In this paper, a finite element model for magnesium alloy stent is studied to simulate the mechanical behavior of the stent. It is composed of $1.5 \mathrm{~mm}$ in inner diameter, $7 \mathrm{~mm}$ length, $80 \mu \mathrm{m}$ thickness and $110 \mu \mathrm{m}$ in cross-sectional width. Six mechanical properties have been studied by mathematical modeling with determination of: (1) stent deployment pressure; (2) the intrinsic elastic recoil of the material used; (3) the stent foreshortening; (4) the stent coverage area, (5) the stent flexibility; and (6) the stress maps.
\end{abstract}

\section{Introduction}

The prospective of temporary, biodegradable intravascular stents has been urged ever since permanent metallic stents had been successfully introduced as an approach to improve the clinical outcome after balloon angioplasty. Therefore, percutaneous transluminal coronary angioplasty (PTCA) assisted with stenting procedure has become a primary treatment to coronary heart disease [1]. Finite element analysis is emerging as one of the most important tools available to material sensitive product developers [2]. Finite element method (FEM) can help discover some valuable mechanical characteristics of stents, arteries, and their interactions, which cannot be easily obtained by normal methods. Recently, a few numbers of studies on the stenting systems by FEM have been stated, such as the balloon-stent analysis by W.walke et al and Linxia Gu et al. [3, 4], the stentplaque-artery analysis by Z. paszenda et al. [5] and the stent-artery analysis by Brand et al. [6]. Their results have depicted that FEM is entirely suitable for evaluating the effects of stent implantation. In our research, pressure is directly applied to the inner surface of the stent and invivo loading condition is considered. The aim of this research is to predict the mechanical properties of the magnesium stent using finite element analysis. In this paper, it is reported that finite element method (FEM) has been applied to simulate the transient expansion process of magnesium stent system to obtain the mechanical properties. Six mechanical properties have been studied by mathematical simulation with determination of: (1) stent deployment pressure; (2) the intrinsic elastic recoil of the material used; (3) the stent foreshortening; (4) the stent coverage area; (5) the stent flexibility; and (6) the stress maps. These results show the potential application and implanted behavior of the Magnesium alloy (MgSnMn) stent design and material. 


\section{Method and Material}

The Pro Engineering parametric modeling tool used to design stent. The inner diameter of the stent is $1.5 \mathrm{~mm}$ and the length is $7.0 \mathrm{~mm}$, whereas, the width and length of the cross-section is $110 \mu \mathrm{m}$ and length is $80 \mu \mathrm{m}$ as shown in figure 1.The stent is assumed to have a uniform internal pressure. Furthermore, since the stent is symmetric about the XY plane, the nodes on the end are constrained by using symmetric boundary conditions. The meshing of the stent is done by using the FEA commercial code Altair Hyper Works version 9. The 3D stent is meshed by using solid 186 tetrahedral elements. Solid186 is higher order 3-D 20-node solid element that exhibits quadratic displacement behavior. The complete stent is meshed with the15161 element with 29774 nodes. The nonlinear isotropic inelastic constitutive response of Magnesium alloy is implemented by a Von Mises plasticity model with isotropic hardening. The Young Modulus is 45GPa; Poisson ratio 0.35 and the yield stress $224 \mathrm{MPa}$. The experimental nonlinear stress vs. strain properties are shown in fig 2.Transient pressure load is applied at the inner surface nodes at the rate of $3.4 \times 10^{-4} \mathrm{~N} . \mathrm{mm}^{-}$ $2 /$ millisecond, whereas, the unloading of the expanded stent occurred at the rate of $6.8 \times 10^{-4} \mathrm{~N} \cdot \mathrm{mm}^{-}$

$2 /$ millisecond. The nonlinear problem has been solved by using a full Newton-Raphson method.

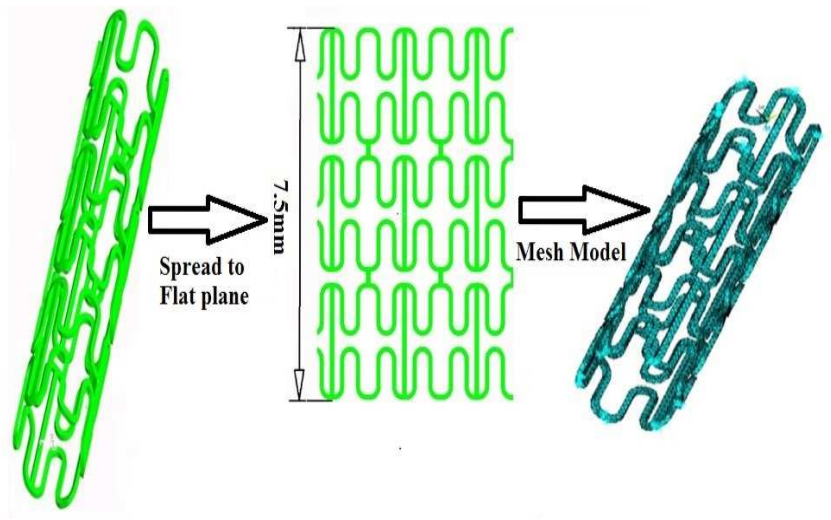

Figure 1 Stent model

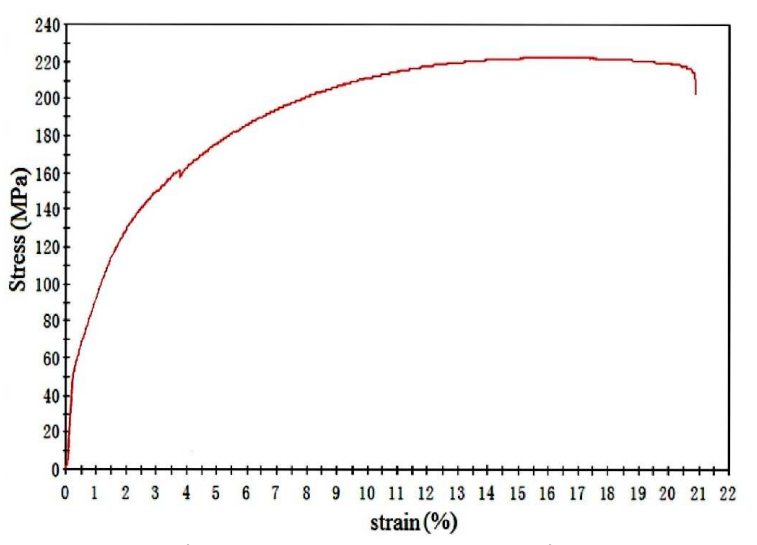

Figure 2 Stress vs. Strain

\section{Results and Discussion}

Stress Analysis. The distribution of residual stress after unloading in the stent is shown in the fig 3. It can be seen from the figure that the highly stressed areas are focalized in the corners of the rings. The maximum residual stress that remained in the stent after deflation is near the constrained area, and it is approximately $164 \mathrm{MPa}$ approaching to the tensile strength $224 \mathrm{MPa}$ of Magnesium alloy. We can observe that the minimum residual stress areas are localized in the middle regions of the cell and middle bridging links.
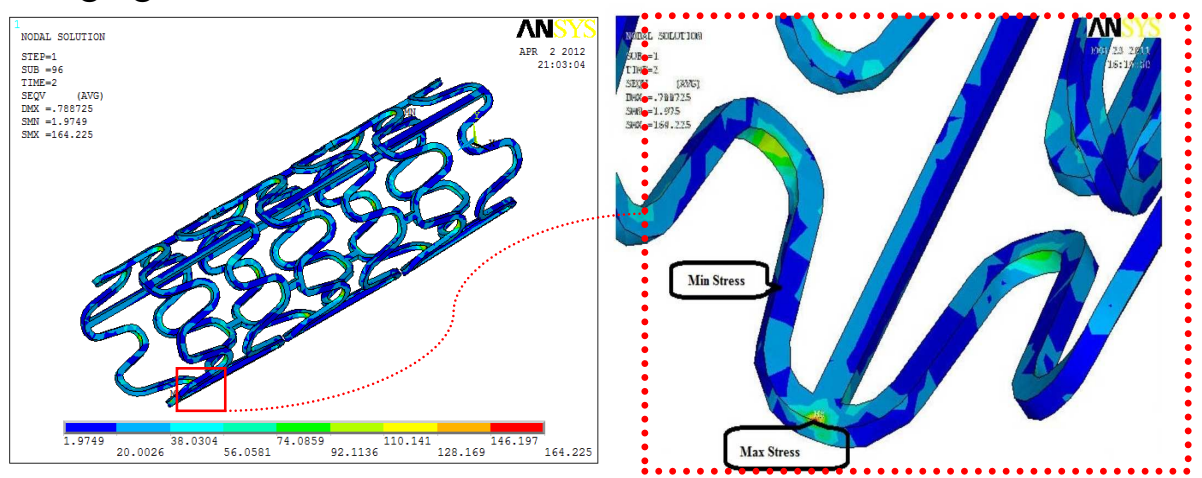

Figure 3 Residual stress in the deformed Stent

Diameter Expansion. Figure 4 shows the distal and central displacement against the pressure at the loading. We can see an expansion of a higher degree in the central area with respect to the distal zones. This is a phenomenon called dog boning characteristic of stent by calculating a ratio as $\frac{D_{\text {distal }}^{\text {load }}-D_{\text {contral }}^{\text {lood }}}{D_{\text {distal }}^{\text {lod }}}$. 

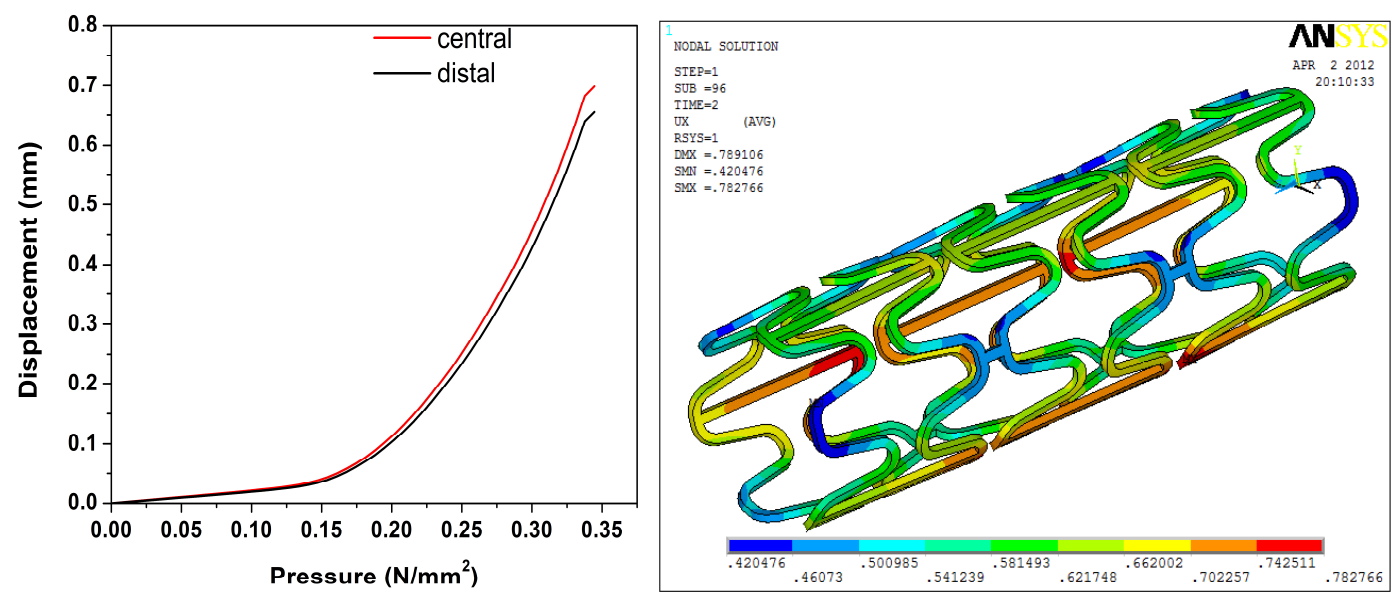

Figure 4 Radial displacement of central and distal node and distribution of displacement

Foreshortening. Foreshortening is defined as the axial displacement of the stent with the pressure loading. Figure 5a shows the maximum axial displacement of node with the deployment pressure. It is seen from the figure that as the deployment pressure increases the foreshortening increases. As the stent diameter increases the foreshortening also increases because it is related to the diameter of the stent. The foreshortening ratio can be calculated by the $\frac{L-L^{\text {load }}}{L}$.

Radial Elastic Recoil. Radial elastic recoil is defined as the ratio of contraction of stent after removing the deployment pressure from the stent. The stent radial strength keeps the stent structure in the deformed shape and prevents it from elastic recoil. Most of the bare metal stent have radial recoil in the range on 2- 5\%. Figure 5b shows the radial recoil of the Magnesium stent. We can see from the figure that upon loading the diameter of the stent continuously increases; and on unloading it reduces its diameter to some extent. The radial recoil can be calculated by $\frac{R^{\text {load }}-R^{\text {unload }}}{R^{\text {load }}}$.
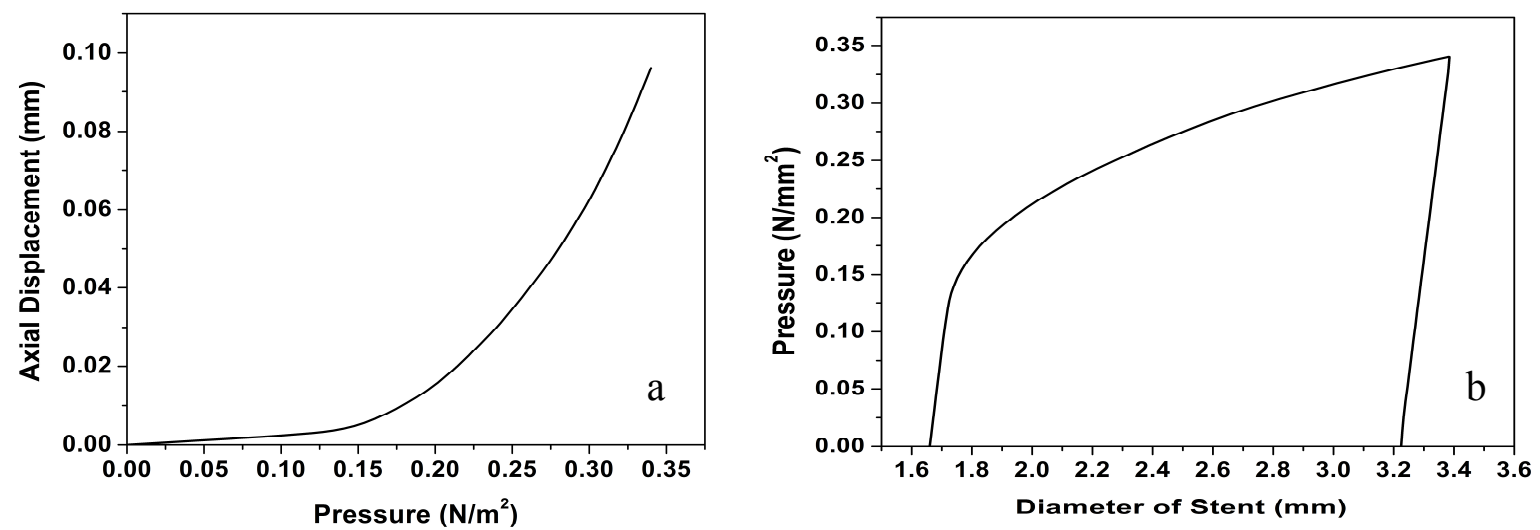

Figure 5 a) Foreshortening curve displacement vs. pressure b) Radial recoil, diameter vs. pressures

Flexibility: Flexibility is required in the stent for better implantation and transportation of the stent on a desired region within the artery. It is evaluated and estimated by applying the force on the middle node of stent. It is found that at $0.013 \mathrm{~N}$ force stent displace $0.6 \mathrm{~mm}$ (shown in fig.6). 

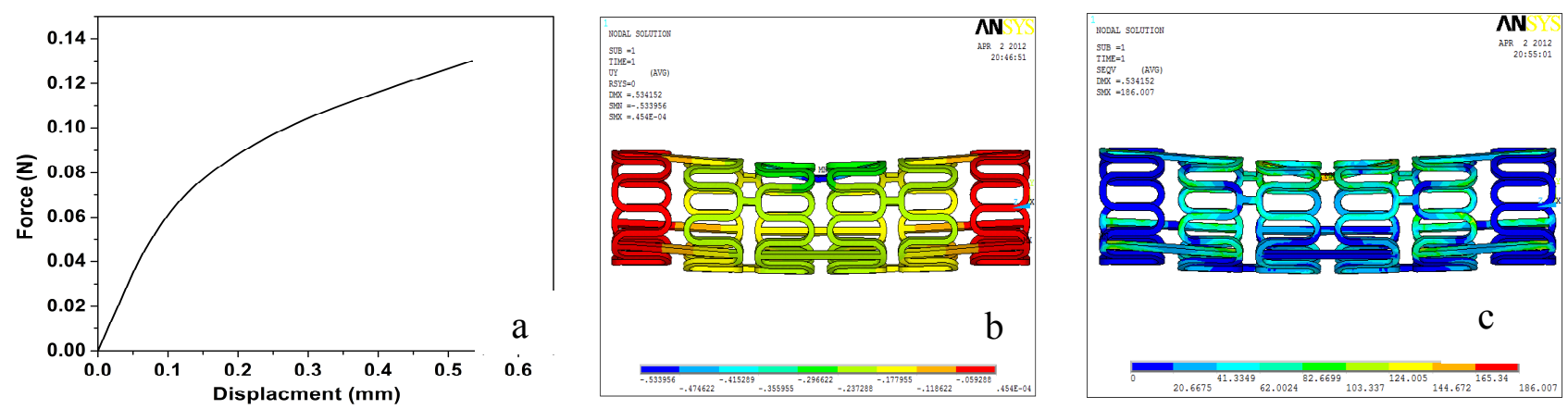

Figure 6 a) Force vs. displacement graph b) Displacement distribution c) Stress Distribution

The above-described characteristics of magnesium alloy stent are summarized in Table 1,

Table 1 Result Summary

\begin{tabular}{lc|lc}
\hline \hline Properties & Values & Properties & Values \\
\hline \hline Dog boning ratio (\%) & -2.934 & Axial recoil ratio (\%) & 0.144 \\
Max Foreshortening ratio (\%) & 1.38 & Deployment pressure (N/mm²) & 0.34 \\
Ultimate foreshortening (\%) & 1.26 & Coverage area (\%) & 15 \\
Radial recoil ratio (\%) & 4.60 & Max stress (MPa) & 164 \\
\hline
\end{tabular}

\section{Conclusion}

Finite element analysis is a technique appropriate to the needs of the medical product designer. The method permits the designer to rapidly access design and to reiterate the design on a computer before a single piece of metal is ever touched. From the finite element analysis results we can conclude that this magnesium alloy stent design have the low radial recoil $4.60 \%$ and better radial stiffness. Besides, it represents uniform expansion with low stresses $164 \mathrm{MPa}$. These results are promising and useful in the study of mechanical performances of the biodegradable stent itself. In particular, they point out the possibility to optimize the flexibility of the magnesium stent by changing bridging links design or by changing the pattern.

\section{Acknowledgement}

This work was supported by a grant from the National High Technology Research and Development Program of China (863 Program, No. 2009AA03Z423). The authors acknowledge the financial support of the Natural Science Foundation of Heilongjiang Province of China (No. 159100120019) and LTCS National Laboratory of China.

\section{Reference}

[1] C. Palmaz, Intravascular stenting: from basic research to clinical application. Cardiovasc. Intervent. Radiol. 15 (1992), pp. 279-284.

[2] F. Migliavacca, L. Petrini, M. Colombo, et al. "Mechanical behavior of coronary stents investigated through the finite element method". Journal of Biomechanics 35 (2002) 803-811

[3] W. Walke, Z. Paszenda and J. Filipiak., Experimental and numerical biomechanical analysis of vascular stent, Journal of Materials Processing Technology, Vol 164-165(2005), pp 12631268.

[4] Linxia Gu, Swadeshmukul Santra, et al, "Finite element analysis of covered microstents", Journal of Biomechanics 38 (2005) 1221-1227.

[5] Z. paszenda, J. Marciniak , Biomechanical characteristics of the stent-coronary vessel system, Acta of Bioengineering and Biomechanics., Vol.4,N0.1,2002

[6] Brand, M,. Ryvkin, The cardiocoil stent-artery interaction. Journal of Biomechanical Engineering Transactions of the ASME 127, 337-344 (2005). 